\title{
On Capacity-Maximizing Angular Densities of Multipath in MIMO Channels
}

\author{
Georgy Levin and Sergey Loyka \\ School of Information Technology and Engineering \\ University of Ottawa \\ 161 Louis Pasteur, Ottawa, Ontario, Canada, K1N 6N5 \\ Tel: (613) 562-5800 ext. 2198, Fax: (613) 562-5175 \\ Email: \{glevin, sloyka\}@site.uottawa.ca
}

\begin{abstract}
This paper provides a partial answer to the question: "what is the best angular density of multipath in MIMO channels?" using the size-asymptotic theory of Toeplitz matrices for uniform 2-D and 3-D antenna arrays. A Kronecker-type approximation of the array correlation structure is proposed and used to find the angular densities that completely eliminate correlation between any elements of antenna arrays and thus maximize the asymptotic MIMO capacity for a broad class of fading distributions. At half-wavelength spacing, the best angular density is shown to be non-uniform, which implies that the popular Clarke's (Jake's) model does not represent the best case scenario. The asymptotic results are validated via Monte-Carlo simulations, and a number of practical guidelines for antenna design and optimal orientation are provided.
\end{abstract}

\section{INTRODUCTION}

There are two major propagation-related factors that affect the capacity of MIMO fading channels: (i) antenna array configuration (e.g. geometry, element spacing), and (ii) the angular density of multipath. A number of capacity optimization problems, which take into account these factors, have been formulated and solved. The impact of angular density on spatial correlation and channel capacity has been discussed in [1]. The effect of fading correlation on the mean/instantaneous capacity and cut-off rate has been studied in [2]. The uniform angular density within a sector has been investigated and the requirements on antenna element spacing have been formulated in [3]. Various models of non-uniform angular densities (e.g. Gaussian, Laplacian, Cosine etc.) have been proposed and validated under various propagation conditions [4].

The question traditionally asked in most of the prior works, in one form or another, is "what is the effect of such-and-such angular density on the channel capacity (correlation, diversity, etc.)?". The only exception is [5], where an opposite question has been posed: "what is the best angular density of multipath in MIMO channels?". While being an intriguing question from a theoretical viewpoint, it is also practicallyrelevant. Specifically, the capacity-maximizing angular density found in [5] for a broad class of MIMO channels with uniform linear arrays (ULA) provided a benchmark for practically existing densities to compare with, as well as led to some practical guidelines for antenna design when the angular density is Gaussian, as adopted by IEEE $802.11 \mathrm{n}$. Best angular density provides an example of a scenario where the antenna array and its electromagnetic environment are matched in information-theoretical sense (capacity-wise).

In the present paper, the problem of the best angular density is solved for a broad class of MIMO channels with uniform 2$\mathrm{D}$ and 3-D antenna arrays. While the relationship between propagation channel characteristics, antenna array design and the channel capacity are rarely amenable to a closed-form analysis, we go around this difficulty by using an asymptotic approximation of the channel capacity (when the number of antennas is large). This allows us to obtain the best angular density in a closed-form for a general class of fading channels (not only Rayleigh/Rice), and also an insight as to why it is of that particular form. The size-asymptotic approach has already been successfully used in a number of works, and has been proven to be reasonably accurate for channels with a moderate number of antennas. For example, asymptotic outage capacity distribution of correlated MIMO Rayleigh-fading channels has been obtained in [6]. The impact of correlation on the asymptotic capacity of unitary-independent-unitary (UIU) channels has been studied in [7]. By letting the number of transmit antennas go to infinity and using circulant matrices theory, an interesting effect of capacity saturation in circular antenna arrays of a fixed aperture has been shown in [8] for the uniformly distributed multipath.

The emphasis of this paper is on information-theoretic rather than electromagnetic analysis of uniform 2-D and 3-D antenna arrays, which also dictates the assumptions we make. In particular, we follow a simplified model of isotropic antenna array elements, which is widely used in the antenna array theory and information-theoretic literature [9]. The main contributions are:

- We show that when the number of antennas is large, the instantaneous and mean capacity of a broad class of MIMO channels (not necessarily Rayleigh-fading) with an arbitrary correlation structure (not necessarily unitary-independentunitary UIU) does not depend on a particular channel distribution, but only on the correlation between antennas. This generalizes/extends the known results obtained earlier for Rayleigh and UIU channels.

- We propose an approximation of multidimensional array correlation structure as a Kronecker product of ULA correlation matrices. The approximation advantages are: (i) it 
is reasonably accurate, (ii) it allows applying the well developed theory of Toeplitz matrices to analysis of multidimensional antenna arrays, and (iii) it is scalable (a 2-D antenna correlation matrix is approximated by the Kronecker product of two ULA correlation matrices, etc.).

- Using the Kronecker-type approximation and Szego Theorem [10], we find the multipath angular density that maximizes capacity of a broad class of MIMO channels with uniform 2-D and 3-D antenna arrays. The capacity-maximizing angular density is shown to be non-uniform, i.e. the popular Clarke's (Jake's) model [11] does not always represent the best-case multipath scenario.

\section{ASYMPTOTIC CAPACITY}

Consider an equivalent base-band discrete model of a MIMO channel with $n_{t}$ Tx and $n_{r}$ Rx antennas

$$
\mathbf{y}=\mathbf{H x}+\mathbf{w} \text {, }
$$

where $\mathbf{x}$ and $\mathbf{y}$ are transmit and receive vectors respectively, $\mathbf{H}$ is the channel matrix whose elements $(\mathbf{H})_{k m}, k=1 \ldots n_{r}$, $m=1 \ldots n_{t}$, represent the complex channel gains from $m^{\text {th }}$ transmit to $k^{\text {th }}$ receive antennas, and $\mathbf{w}$ is the AWGN noise vector. We adopt the following assumptions: (i) the channel state information (CSI) is available at the Rx end but not at the Tx end, (ii) $\mathbf{x} \propto \mathcal{C N}\left(\mathbf{0}, P_{T} / n_{t} \mathbf{I}\right){ }^{1}$, where $\propto$ means identically distributed, I is identity matrix, and $P_{T}$ is the total transmitted power, which does not depends on $n_{t}$ (this achieves the ergodic capacity of the i.i.d. Rayleigh fading channel [1], and is a reasonable transmission strategy with no Tx CSI in general), (iii) $\mathbf{w} \propto \mathcal{C N}\left(\mathbf{0}, N_{0} \mathbf{I}\right)$, where $N_{0}$ is the noise variance in each receive antennas, (iv) the channel is frequency flat and quasi-static (slow block fading).

The instantaneous capacity $C$ (i.e. the capacity of a given channel realization) and mean capacity $\bar{C}$ per Rx antenna of the MIMO channel in natural units $[$ nat $]$ are [1]

$$
C=n_{r}^{-1} \ln \operatorname{det}\left[\mathbf{I}+\gamma_{0} / n_{t} \cdot \mathbf{H H}^{H}\right], \bar{C}=E(C),
$$

where operator $E$ denotes expectation and $\gamma_{0}$ is the SNR per $\mathrm{Rx}$ antenna. Without loss of generality $\mathbf{H}$ is normalized so that $E\left\{\|\mathbf{H}\|^{2}\right\}=n_{t} n_{r}$, where \|\| is Frobenius norm. The transmit and receive correlation matrices are defined as $\mathbf{R}_{r}=n_{t}^{-1} E\left\{\mathbf{H H}^{H}\right\}$ and $\mathbf{R}_{t}=n_{r}^{-1} E\left\{\mathbf{H}^{H} \mathbf{H}\right\}$ respectively. Due to the adopted normalization $\operatorname{tr}\left\{\mathbf{R}_{r}\right\}=n_{r}$ and $\operatorname{tr}\left\{\mathbf{R}_{t}\right\}=n_{t}$, where $t r$ stands for trace. The following theorem gives asymptotic instantaneous and mean capacity of the MIMO channel when $n_{t}$ and $n_{r}$ go to infinity.

Theorem 1: Let $\mathbf{H}$ be a complex circular symmetric random matrix (not necessarily Gaussian, i.i.d. or with a separable correlation structure as in [6], [7]), and the following conditions are satisfied as $n_{t}, n_{r} \rightarrow \infty$ :

$$
\text { (i) } n_{t}^{-2} \sum_{k, m=1}^{n_{t}} \sum_{n, l=1}^{n_{r}} \kappa_{4}\left(H_{n m} H_{n k} H_{l k} H_{l m}\right) \rightarrow 0
$$

where $\kappa_{4}\left(H_{n m} H_{n k} H_{l k} H_{l m}\right)$ is the fourth order cumulant of circular symmetric random variables.

\footnotetext{
${ }^{1}$ In practice this assumption corresponds to capacity-approaching codes.
}

(ii) $\frac{1}{n_{t}^{2}}\left(\left(\sum_{k, l=1}^{n_{t}}\left|\operatorname{tr}\left\{\mathbf{Q}_{k l}\right\}\right|^{2}\right)^{1 / 2}+\left(\sum_{k, l=1}^{n_{r}}\left|\operatorname{tr}\left\{\mathbf{G}_{k l}\right\}\right|^{2}\right)^{1 / 2}\right)-\left\|\mathbf{R}_{r}\right\|^{2} \rightarrow 0^{2}$, (4)

where $\mathbf{Q}_{k l}=E\left\{\mathbf{h}_{k} \mathbf{h}_{l}^{H}\right\}$ and $\mathbf{G}_{k l}=E\left\{\mathbf{g}_{k} \mathbf{g}_{l}^{H}\right\}, \mathbf{h}_{k}$ and $\mathbf{g}_{k}$ are the $k^{\text {th }}$ columns of $\mathbf{H}$ and $\mathbf{H}^{H}$ respectively.

As $n_{t} \rightarrow \infty$, the capacity per Rx antenna converges as

$$
\text { (a) } C \stackrel{p}{\rightarrow} C^{*} ; \text { (b) } \bar{C} \rightarrow C^{*},
$$

where $C^{*}=n_{r}^{-1} \ln \operatorname{det}\left[\mathbf{I}+\gamma_{0} \mathbf{R}_{r}\right]$, and $\stackrel{p}{\rightarrow}$ denotes convergence in probability.

Proof: Main steps to prove (a) are given in [5]. A proof of (b) follows from (a) using Jensen inequality ${ }^{3}$.

Theorem 1 allows splitting the effect of correlation at the transmit and receive ends and indicates that in asymptotic approximation the channel capacity does not depend on a particular distribution of $\mathbf{H}$, or $\mathbf{R}_{t}$, but only on $\mathbf{R}_{r}$. Since the condition (4) does not hold when the Tx end is fully correlated [5], which corresponds to small antenna spacing, we conjecture that (4) is satisfied if the antenna spacing exceeds a certain minimum. Note that $C^{*}$ is the upper bound on the mean capacity of MIMO channels with a finite number of antennas $\left(\bar{C} \leq C^{*}\right)$ [3], i.e. following Theorem 1, this bound is asymptotically tight. Conditions (3) and (4) are elaborated in detail in [5]. It can be shown that as special cases Theorem 1 includes a number of popular channel models such as i.i.d. and correlated Rayleigh/Rice, UIU channels for which the results in (5) are known, see e.g. [6], [7]. In particular, if $\mathbf{H}$ is i.i.d. complex circular symmetric Gaussian, it is straightforward to show that Theorem 1 holds if $\lim _{n_{t}, n_{t} \rightarrow \infty} n_{r} / \sqrt{n_{t}}=0$, i.e. $n_{t}$ has to increase much faster than $n_{r}$.

Theorem 1 will be used later on to find the best multipath angular density in terms of channel capacity. However, in order to proceed toward this goal, an approximation of the antenna array correlation structure is proposed in the next section.

\section{KRONECKER-TYPE APPROXIMATION}

Consider a uniform rectangular array (URA) lying on the $X Y$ plane with $n_{x}$ and $n_{y}$ antennas along $x$ and $y$ coordinates respectively, so that $n_{r}=n_{x} \cdot n_{y}$. Assume that the correlation between the antenna elements along $x$ coordinate does not depend on $y$ and is given by matrix $\mathbf{R}_{x}$, and the correlation along $y$ coordinate does not depend on $x$ and is given by matrix $\mathbf{R}_{y}$. The following Kronecker-type approximation of the URA correlation matrix is proposed:

$$
\mathbf{R}_{r}=\mathbf{R}_{x} \otimes \mathbf{R}_{y},
$$

where $\otimes$ denotes the Kronecker product. Following (6), zero correlation between two antennas $A_{1}$ and $A_{2}$ located in a row (along $x$ or $y$ coordinate), enforces zero correlation between $A_{1}$ and all the antenna elements in the column containing $A_{2}$, e.g. if $\left(\mathbf{R}_{x}\right)_{k, m}=0$, where $\left(\mathbf{R}_{x}\right)_{k, m}$ is the $k, m$ element of

\footnotetext{
${ }^{2}$ We show later a case when this condition implies that $n_{t}$ has to increase much faster than $n_{r}$.

${ }^{3}$ In general convergence in the mean implies convergence in probability, but not vise versa [12].
} 
$\mathbf{R}_{x}$, then the whole block $\left(\mathbf{R}_{r}\right)_{i, j}=0$, $i=(k-1) n_{x}+1, \ldots, k n_{x}, \quad j=(m-1) n_{y}+1, \ldots, m n_{y}$. From (6),

$\mathbf{R}_{r}$ is the Kronecker product of two ULA correlation matrices $\mathbf{R}_{x}$ and $\mathbf{R}_{y}$, which are Toeplitz ${ }^{4}$. Therefore, even though $\mathbf{R}_{r}$ may not be Toeplitz, its approximation (6) has a Toeplitz structure.

To assess the accuracy of the Kronecker-type approximation we use the scalar measure of correlation $n_{r}^{-1}\left\|\mathbf{R}_{r}\right\| \in\left[1 / \sqrt{n_{r}}, 1\right]^{5}$ that accounts for total correlation between multiple antennas and simultaneously affects the diversity measure and outage capacity distribution of MIMO channels when a number of antennas is large [13], [14]. $n_{r}^{-1}\left\|\mathbf{R}_{r}\right\|=1 / \sqrt{n_{r}}$ corresponds to a case where the antennas are completely uncorrelated, i.e. $\mathbf{R}_{r}=\mathbf{I}$, for fully correlated antennas $n_{r}^{-1}\left\|\mathbf{R}_{r}\right\|=1$, i.e. $\mathbf{R}_{r}$ has a single non-zero eigenvalue equal $n_{r}$. The higher the $n_{r}^{-1}\left\|\mathbf{R}_{r}\right\|$, the lower the outage capacity at outage probabilities $<0.5$ [14]. From (6)

$$
n_{r}^{-1}\left\|\mathbf{R}_{r}\right\|=n_{x}^{-1}\left\|\mathbf{R}_{x}\right\| \cdot n_{y}^{-1}\left\|\mathbf{R}_{y}\right\|,
$$

i.e. under the Kronecker-type approximation the measure of correlation of the $\mathrm{Rx}$ antenna is the product of the corresponding measures of $\mathbf{R}_{x}$ and $\mathbf{R}_{y}$.

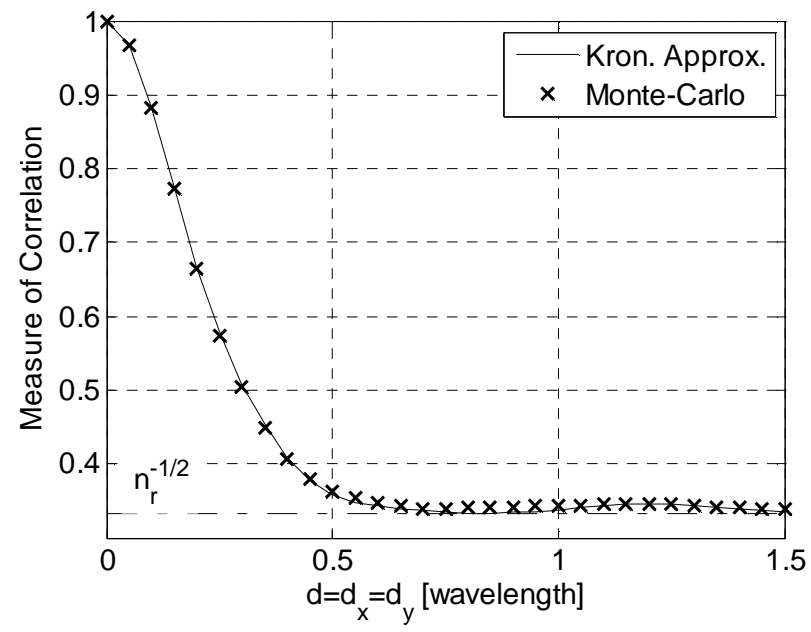

Fig. 1 Measure of correlation of 3x3 URA vs. antenna spacing. Uniformly distributed multipath.

Consider a URA in a uniformly distributed 3-D multipath. It is straightforward to show that in this case the Toeplitz matrix-generating vectors ${ }^{6}$ of $\mathbf{R}_{x}$ and $\mathbf{R}_{y}$ are the same and given by

$$
R_{k}=J_{0}^{2}(\pi k d),
$$

where $J_{0}(x)$ is zero-order Bessel function of the first kind, and $d$, either $d_{x}$ or $d_{y}$, is the antenna spacing in wavelengths along $x$ or $y$ coordinates respectively. Fig. 1 shows the measure of correlation of a $3 \times 3$ URA vs. antenna spacing $d=d_{x}=d_{y}$. The solid line is calculated analytically using (6)

\footnotetext{
${ }^{4}$ Toeplitz correlation matrix physically corresponds to uniform antenna array geometry, when correlation depends on the spacing between elements only, but not on their positions.

${ }^{5}$ The measure applies to a set of normalized correlation matrices such that $t r \mathbf{R}_{r}=n_{r}$.

${ }^{6}$ The generating vector of an $n \times n$ Toeplitz matrix $\mathbf{R}$ is defined as $R_{k-m}=(\mathbf{R})_{k m}, k, m=1 \ldots n$, where $(\mathbf{R})_{k m}$ is an element of $\mathbf{R}[10]$.
}

and (8). The dotted line is obtained via Monte-Carlo simulation, when the multipath is uniformly distributed. It can be seen that the main lobe $(d<0.5)$ is well approximated by (6), while there is small discrepancy on the tail $(d \geq 0.5)$. At $d \approx 0.75$, the measure of correlation is very close to the minimum $n_{r}^{-1 / 2}=1 / 3$, which corresponds to an uncorrelated URA.

The following corollary is based on Theorem 1 and utilizes the Kronecker-type approximation of URA correlation structure.

Corollary 1: Consider a MIMO channel that satisfies the conditions of Theorem 1 and whose correlation matrix $\mathbf{R}_{r}=\mathbf{R}_{x} \otimes \mathbf{R}_{y}$ has a Toeplitz structure. Let $\mathbf{R}_{r}$ be nondegenerate and square-summable ${ }^{7}$, i.e. $0<\sum_{k=-\infty}^{\infty}\left|R_{k}\right|^{2}<\infty$, where $R_{k}$ is the generating vector of $\mathbf{R}_{r}$. As $n_{x}, n_{y}$ and $n_{t}$ go to infinity, the channel capacity per Rx antenna converges as

$$
\bar{C} \rightarrow(2 \pi)^{-2} \int_{-\pi}^{\pi} \int_{-\pi}^{\pi} \ln \left[1+\gamma_{0} \lambda_{x}(u) \cdot \lambda_{y}(v)\right] d u d v,
$$

where $\lambda_{x}(u)$ and $\lambda_{y}(v), u, v \in(-\pi ; \pi]$ are the spectrums of $\mathbf{R}_{x}$ and $\mathbf{R}_{y}$ respectively $^{8}$.

Proof: Due to Szego Theorem [10], using the fact that the eigenvalues of $\mathbf{R}_{r}$ are given by the product of the corresponding eigenvalues of $\mathbf{R}_{x}$ and $\mathbf{R}_{y}$.

Maximum Asymptotic Capacity: It is straightforward to show using Hadamard inequality that $0 \leq \operatorname{det}(\mathbf{R}) \leq 1$ for any $n \times n$ correlation matrix $\mathbf{R}$ normalized such that $\operatorname{tr}(\mathbf{R})=n$. Therefore, the asymptotic capacity in (5) is maximal when $\mathbf{R}_{r}=\mathbf{I}$, i.e. the channel is uncorrelated at the Rx end. Using Kronecker approximation (6), this implies that both $\mathbf{R}_{x}=\mathbf{I}$, $\mathbf{R}_{y}=\mathbf{I}$ with corresponding spectrums

$$
\lambda_{x}(u)=\lambda_{y}(u)=1, u \in(-\pi ; \pi]
$$

From (9) and (10), the channel maximal capacity per Rx antenna is $C_{\max }=\ln \left(1+\gamma_{0}\right)$, i.e. in the asymptotic approximation, each additional $\mathrm{Rx}$ antenna may increase the total capacity by the amount not beyond the capacity of an $1 \mathrm{x} 1$ AWGN channel. The multipath angular density that achieves the maximum $C_{\max }$ for a broad class of MIMO channels with uniform 2-D and 3-D antenna arrays is derived in the following sections.

\section{Best ANGULAR DENSITY FOR URA}

Consider a MIMO channel in a 3-D multipath environment. Let $R(x, y)$ denote the spatial correlation between two antennas at spacing $\sqrt{x^{2}+y^{2}}$. The multipath wave-number spectrum and the correlation function are related by the Fourier Transform [9]

$$
f\left(k_{x}, k_{y}\right)=(2 \pi)^{-2} \int_{-\infty}^{\infty} \int_{-\infty}^{\infty} R(x, y) e^{-j\left(k_{x} \cdot x+k_{y} \cdot y\right)} d x d y,
$$

${ }^{7}$ If $\mathbf{R}_{r}$ is non-degenerate and absolutely summable, ${ }_{2}$ it also satisfies the condition of Corollary 1, since $\sum_{n=-\infty}^{\infty}\left|R_{n}\right|^{2} \leq\left(\sum_{n=-\infty}^{\infty}\left|R_{n}\right|\right)^{2}$.

${ }^{8}$ The spectrum of a matrix $\mathbf{R}$ is given by $\lambda(u)=\sum^{\infty=-\infty} R_{k} e^{j n u}$, where $R_{k}$ is the generating vector of $\mathbf{R}$ and $j=(-1)^{1 / 2}[10]$. 
where $k_{x}$ and $k_{y}$ are $x$ and $y$ components of the wavevector $\mathbf{k} . f\left(k_{x}, k_{y}\right)$ is often referred as the joint probability density function (PDF) of $k_{x}$ and $k_{y}$ due to the following properties [9]: (i) $f\left(k_{x}, k_{y}\right)$ is real and non-negative assuming that $R(x, y)$ is Hermitian for both $x$ and $y$, (ii) under normalization $R(0,0)=1, \int_{-\infty}^{\infty} \int_{-\infty}^{\infty} f\left(k_{x}, k_{y}\right) d k_{x} d k_{y}=1$.

Consider now a receiving URA of isotropic antenna elements, when the correlation between the elements is given by a Toeplitz matrix $\mathbf{R}_{r}=\mathbf{R}_{x} \otimes \mathbf{R}_{y}$ (as suggested by approximation (6)). In this case

$$
R_{x, n}=R\left(d_{x} n, 0\right), R_{y, n}=R\left(0, d_{y} n\right), n=0,1,2 \ldots,
$$

where $R_{x, n}$ and $R_{y, n}$ are the Toeplitz matrix-generating vectors of $\mathbf{R}_{x}$ and $\mathbf{R}_{y}$ respectively. It is straightforward to show that due to (6) $R(x, y)$ can be thus factorized as $R(x, y)=R(x, 0) R(0, y)$, so that using (11)

$$
f\left(k_{x}, k_{y}\right)=f_{x}\left(k_{x}\right) f_{y}\left(k_{y}\right),
$$

i.e. under the Kronecker approximation, $k_{x}$ and $k_{y}$ are independent.

From the geometry of the problem, the link between $k_{x}$, $k_{y}$ and the angles of arrival of multipath components is $\psi_{x}=k_{x} d_{x}=2 \pi d_{x} \sin \theta \cos \phi, \quad \psi_{y}=k_{y} d_{y}=2 \pi d_{y} \sin \theta \sin \phi$, where $\theta \in[0 ; \pi]$ and $\phi \in[0 ; 2 \pi)$ are the elevation and azimuth angles, $\psi_{x}, \psi_{y}$ represent the phase difference between two adjacent antennas along $x$ and $y$ coordinates respectively. From (12) $R_{x, n}$ and $R_{y, n}$ are the samples of the continuous function $R(x, y)$, hence the relationship between the spectrums $\lambda_{x}(u), \lambda_{y}(u)$ of $\mathbf{R}_{x}, \mathbf{R}_{y}$ and the spectrum $f\left(k_{x}, k_{y}\right)$ of $R(x, y)$ is given by the sampling theorem,

$$
\begin{aligned}
& \lambda_{x}(u)=2 \pi \sum_{n=-\infty}^{\infty} f_{\psi_{x}}(u-2 \pi n) \\
& \lambda_{y}(u)=2 \pi \sum_{n=-\infty}^{\infty} f_{\psi_{y}}(u-2 \pi n)
\end{aligned}
$$

where $f_{\psi_{x}}(u)=f_{x}\left(u / d_{x}\right) / d_{x}, \quad f_{\psi_{y}}(u)=f_{y}\left(u / d_{y}\right) / d_{y}$ are the PDF's of $\psi_{x}$ and $\psi_{y}$ respectively. Therefore, under the conditions of Theorem 1 and following Corollary 1 , one obtains the condition to achieve the maximal capacity $C_{\max }$ by substituting (10) in (14): $\bar{C} \rightarrow C_{\max }$ if

$$
\begin{aligned}
& 2 \pi \sum_{n=-\infty}^{\infty} f_{\psi_{x}}(u-2 \pi n)=1 \\
& 2 \pi \sum_{n=-\infty}^{\infty} f_{\psi_{y}}(u-2 \pi n)=1
\end{aligned}, u \in(-\pi ; \pi]
$$

from which we obtain the following:

Theorem 2: Consider a MIMO channel equipped with a URA at the Rx end. Under the conditions of Theorem 1, as both $n_{t}$ and $n_{r}$ go to infinity, the following holds:

(i) if either $d_{x}$ or $d_{y}<1 / 2$, there are no such $f_{\psi_{x}}(u)$, $f_{\psi_{y}}(u)$ that $\bar{C} \rightarrow C_{\max }$, i.e. the upper bound is not achievable.

(ii) if both $d_{x}, d_{y} \geq 1 / 2$, there is a class of $f_{\psi_{x}}(u), f_{\psi_{y}}(u)$ such that $\bar{C} \rightarrow C_{\max }$, i.e. the maximizing $f_{\psi_{x}}(u), f_{\psi_{y}}(u)$ are not unique.

(iii) if $d_{x}=d_{y}=1 / 2, \quad \bar{C} \rightarrow C_{\max }$ for $\psi_{x}$ and $\psi_{y}$ distributed uniformly, i.e. $f_{\psi_{x}}(u)=f_{\psi_{y}}(u)=(2 \pi)^{-1}$. The corresponding multipath angular density is "Sine" distributed

$$
f_{\theta, \phi}(\theta, \phi)=\frac{1}{4 \pi} \sin (\theta), \theta \in[0 ; \pi], \phi \in[0 ; 2 \pi)
$$

and the corresponding spatial correlation function is

$$
R(x, y)=\operatorname{sinc}(2 x) \operatorname{sinc}(2 y),
$$

where $\operatorname{sinc}(x)=\sin (\pi x) /(\pi x)$.

Proof: Using (15) and Jacobian transformation from Cartesian to spherical coordinates.

Theorem 2 suggests that when $d_{x}=d_{y}=1 / 2$, the best multipath angular density $f_{\theta, \phi}(\theta, \phi)$ is non-uniform in terms of $\theta$, and circular symmetric in terms of $\phi$. The latter is well explained by the rotational symmetry of the URA with an asymptotically large number of antennas. Furthermore, since the marginal distribution of $\phi$ is uniform, it follows that in the $d_{x}=d_{y}=1 / 2$ case the elevation and azimuth angles are independent.

As a particular case of statement (ii) of Theorem 2, it is straightforward to show using (14), that for any continuous multipath angular density with no specular components (no Dirac's delta functions in the density), (15) always holds true as $d_{x}, d_{y} \rightarrow \infty$, and hence $\bar{C} \rightarrow C_{\max }$.

As a general remark, we note that when a given angular density eliminates correlation between any two elements of a URA of spacing $\sqrt{x^{2}+y^{2}}$, it also does so for spacing $\sqrt{(n x)^{2}+(m y)^{2}}$, where $n$ and $m$ are integers.

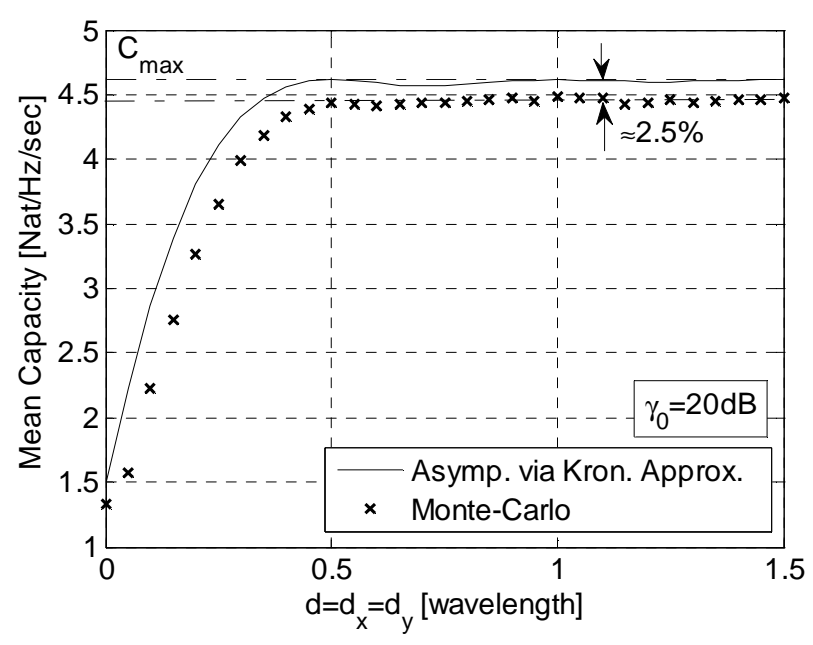

Fig. 2 Mean capacity per Rx antenna vs. antenna spacing. $4 x 4$ Tx URA, 2x2 Rx URA. "Sine" distributed multipath (16).

Even though Theorems 1 and 2 are based on the sizeasymptotic assumptions and the Kronecker-type approximation, simulations show that their results apply to the channels with a finite (moderate) number of antennas, and therefore practically relevant. As an example, Fig. 2 shows the mean capacity per $\mathrm{Rx}$ antenna vs. antenna spacing $d=d_{x}=d_{y}$. The solid line is calculated analytically using (5b) and (17). The dotted line is obtained via Monte-Carlo simulation of a MIMO Rayleigh-fading channel with $4 \times 4$ transmitting and $2 \times 2$ receiving URA's when the multipath at both ends is distributed according to (16). It can be seen that the mean and asymptotic capacities well coincide, the discrepancy does not exceed $2.5 \%$ of the capacity maximum even though the simulated MIMO channel has a moderate 
number of antennas. Moreover, the asymptotic theory predicts well the behavior of the mean capacity. In both asymptotic and finite cases the capacity maxima occur at $d=n / 2, n=1,2, \ldots$, where the channel becomes uncorrelated. Therefore, even though (16) is obtained under the asymptotic assumptions, it is still the best angular density in terms of the mean capacity when the number of antennas is finite.

Simultaneous analysis of Figs. 1 and 2 shows that the rate of correlation decreasing or capacity increasing with antenna spacing depends very much on the angular density. The rate is faster when the density follows (16) as compared to the uniform one. Particularly, in the former case the channel becomes uncorrelated (achieves capacity maximum) at $d=0.5$, while in the latter this happens at $d \approx 0.75$. Thus the Clarke's (Jake's) model [11], where $f_{\theta, \phi}(\theta, \phi)$ is assumed to be uniform, does not represent the best case scenario when $d_{x}=d_{y}=1 / 2$.

\section{BeSt ANGULAR DENSITY FOR UCA}

Consider a uniform cubic array (UCA) of receive antenna elements in a 3-D multipath environment. As in the URA case, assume that the correlation between the antenna elements along $x$ coordinate does not depend on $y$ and $z$, and is given by matrix $\mathbf{R}_{x}$, the correlation along $y$ coordinate does not depend on $x$ and $z$, and is given by matrix $\mathbf{R}_{y}$ and the correlation along $z$ coordinate does not depend on $x$ and $y$, and is given by matrix $\mathbf{R}_{z}$. Applying the same concept as for the URA, the Kronecker-type approximation of the UCA correlation matrix is

$$
\mathbf{R}_{r}=\mathbf{R}_{x} \otimes \mathbf{R}_{y} \otimes \mathbf{R}_{z}
$$

From the geometry of the problem the link between the components of the wave-vector $\mathbf{k}: k_{x}, k_{y}, k_{z}$ and the angles of arrival of multipath is $\psi_{x}=k_{x} d_{x}=2 \pi d_{x} \sin \theta \cos \phi$, $\psi_{y}=k_{y} d_{y}=2 \pi d_{y} \sin \theta \sin \phi$, and $\psi_{z}=k_{z} d_{z}=2 \pi d_{z} \cos \theta$, where $d_{x}, d_{y}$ and $d_{z}$ are the antenna element spacing in wavelengths along $x, y$ and $z$ coordinates respectively. Let $f_{\psi_{x}}(u), f_{\psi_{y}}(u)$ and $f_{\psi_{z}}(u)$ be the marginal PDF's of $\psi_{x}$, $\psi_{y}$ and $\psi_{z}$, which can be proven to be independent under approximation (18). Adding $z$ coordinate and applying the same arguments as for the URA we obtain the following:

Theorem 3: Consider a MIMO channel equipped with a UCA at the Rx end. Under the conditions of Theorem 1 and for $d_{x}=d_{y}=d_{z}=1 / 2, \bar{C} \rightarrow C_{\max }$ as both $n_{t}$ and $n_{r}$ go to infinity, when $\psi_{x}, \psi_{y}$ and $\psi_{z}$ are uniformly distributed, i.e. $f_{\psi_{x}}(u)=f_{\psi_{y}}(u)=f_{\psi_{z}}(u)=(2 \pi)^{-1}$. The corresponding multipath angular density is non-uniform and given by (16).

Proof: following the same arguments as for Theorem 2 .

The fact that the best multipath angular density (16) is nonuniform has certain practical implications. Consider, for example, a 3-D environment, where the multipath is not isotropic, but concentrated around a horizontal plane. Theorems 2 and 3 suggest that regardless of any specifics of the system design, the URA and UCA should be mounted in parallel to that plane in order to increase the capacity. We note that following the discussion in Section II, this guideline holds for a broad class of MIMO channels, not necessarily Rayleighfading.

\section{CONCLUSION}

It is of particular interest to search propagation scenarios, where the multipath distribution fits the best angular density. In such scenarios the antenna array and the multipath would be matched in a probabilistic sense providing maximal MIMO capacity. In addition, the best $f_{\theta, \phi}(\theta, \phi)$ can be used as a theoretical benchmark indicating, for example, how far away a given angular density is from the best one, which, in turn, can provide useful tips on optimal antenna spacing and array orientation for practically existing propagation environments.

\section{REFERENCES}

[1] I. E. Telatar, "Capacity of Multi-Antenna Gaussian Channels", $A T \& T$ Bell Labs, Internal Tech. Memo, pp. 1-28, June 1995, (European Trans. Telecom., v.10, no. 6, pp. 585-595, Dec. 1999).

[2] M. T. Ivrlac, W. Utschick, J. A. Nossek, "Fading Correlations in Wireless MIMO Communication Systems," IEEE Journal on Selected Areas in Communications, vol.21, no.5, pp. 819-828, June 2003.

[3] S. Loyka, G. Tsoulos, "Estimating MIMO System Performance Using the Correlation Matrix Approach," IEEE Communications Letters, vol. 6, no. 1, pp. 19-21, Jan 2002.

[4] K. H. Li, M.A Ingram, A. V. Nguyen, "Impact of Clustering in Statistical Indoor Propagation Models on Link Capacity," IEEE Transactions on Communications, vol.50, no.4, pp.521-523, Apr. 2002

[5] G. Levin, S. Loyka, "What is the Best Angular Density of Multipath in MIMO Channels?", in Proc. Canadian Workshop on Information Theory (CWIT2009), Ottawa, ON, May 2009.

[6] C. Martin, B. Ottersten, "Asymptotic Eigenvalue Distributions and Capacity for MIMO Channels under Correlated Fading," IEEE Transactions on Wireless Communications, vol.3, no.4, pp. 1350-1359, July 2004.

[7] A. M. Tulino, A. Lozano, S. Verdu, "Impact of Antenna Correlation on the Capacity of Multiantenna Channels," IEEE Transactions on Information Theory, vol.51, no.7, pp. 2491-2509, July 2005.

[8] T. S. Pollock, T. D. Abhayapala, R. A. Kennedy, "Antenna Saturation Effects on Dense Array MIMO Capacity", in Proc. ICASSP 2003, IEEE International Conference on Acoustics, Speech, and Signal Processing, vol.4, no. 6-10, pp. IV-361-4, 2003.

[9] H. L. Van-Trees, Optimum Array Processing: Part IV of Detection, Estimation, and Modulation Theory, John Wiley \& Sons, Inc., NY, 2002.

[10] R. M. Gray, "Toeplitz and Circulant Matrices: A Review", Foundations and Trends in Commun. and Inform. Theory, vol. 2, no 3, pp. 155-239, 2006.

[11] W. C. Jakes, Microwave Mobile Communications, Willey, NY, 1974.

[12] T. S. Ferguson, A Course in Large Sample Theory, Chapman \& Hall/CRC, $1^{\text {st }}$ Ed. Reprint, 2002.

[13] M. T. Ivrlac, J. A. Nossek, "Quantifying Diversity and Correlation in Rayleigh Fading MIMO Communication Systems," Signal Processing and Information Technology, 2003. ISSPIT 2003. Proceedings of the 3rd IEEE International Symposium on, pp. 158-161, 14-17 Dec. 2003.

[14] G. Levin, S. Loyka, S., "On the Outage Capacity Distribution of Correlated Keyhole MIMO Channels", IEEE Trans. on Inform. Theory, vol.54, no.7, pp.3232-3245, July 2008. 